\title{
Determination of the Dielectric Properties of Ceria and Soot Powders by the Microwave Cavity Perturbation Method
}

\author{
Stefanie Walter ${ }^{1}$, Carsten Steiner ${ }^{1}$, Gunter Hagen ${ }^{1}$, Ralf Moos ${ }^{1}$ \\ ${ }^{1}$ Department of Functional Materials, University of Bayreuth, 95447 Bayreuth, Germany \\ functional.materials@uni-bayreuth.de
}

\begin{abstract}
Summary:
A comprehensive method to determine dielectric properties using the microwave cavity perturbation method (MCP) is presented. It is applicable to a wide range of sample geometries and properties. First tests with typical materials for exhaust aftertreatment have already been carried out successfully. The application of the method can provide further knowledge for radio frequency-based analysis of catalysts and filter systems.
\end{abstract}

Keywords: radio frequency $(\mathrm{RF})$, microwave cavity perturbation, material characterization, dielectric properties, depolarization

\section{Introduction}

In order to meet the increasingly stringent emission standards, the application of automotive exhaust gas aftertreatment systems is essential. To ensure correct operation, knowledge of the state of a catalytic converter or the soot loading of a filter is necessary. A method suitable for this purpose is the radio frequency (RF)-based state diagnosis. This measuring system is based on the change in the dielectric properties of the catalyst or filter depending on their current state. The applicability of this system has already been shown in several studies [1,2].

In order to optimize the technical application of this measuring approach, a deeper understanding of the electrical properties of the used materials in exhaust gas aftertreatment systems is necessary. Recently, a test bench was presented, that enables the characterization of small amounts of powder materials under defined conditions using the microwave cavity perturbation (MCP) method [3].

The exact calculation of the material properties for the investigated samples using the resonance properties is not trivial, since often not all assumptions of the classical microwave cavity perturbation theory (MCPT) are fulfilled. While in previous publications a simplified approach was sufficient to investigate the relative change of the polarization $\varepsilon_{r}{ }^{\prime}$ and the dielectric losses $\varepsilon_{r}{ }^{\prime \prime}$, this work aims to show, using the example of ceria and soot, that a more precise determination of material properties is possible with an extended method.

\section{Microwave Cavity Perturbation}

The determination of the material parameters by the MCP is based on the change of the resonant mode properties due to the introduction of a small material sample into the resonance cavity. The setup shown in Fig. 1 was designed to ensure that the $\mathrm{TM}_{010}$ and $\mathrm{TM}_{020}$ modes have a constant electric field along the resonator axis and thus in the sample area [3].

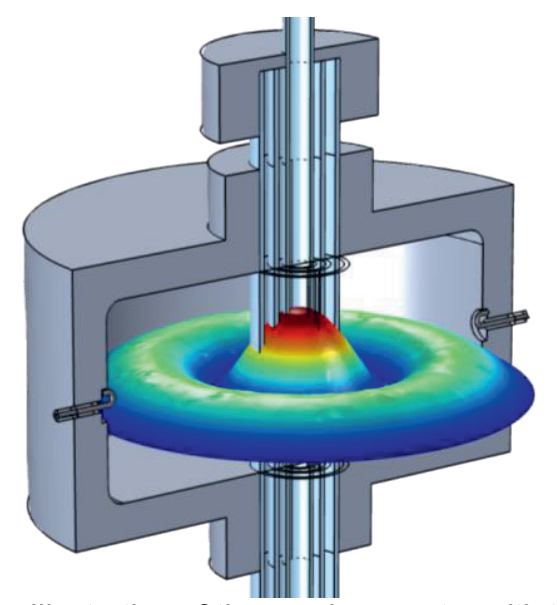

Fig. 1. Illustration of the used resonator with the electric field distribution of the TM020 mode.

In the simplified MCP theory, the material polarization $\varepsilon_{r}{ }^{\prime}$ can be calculated from the shift of the resonant frequency $\Delta f$ due to the sample, the sample volume $V_{S}$ and an effective resonator volume $V_{\text {eff }}$, that is dependent on the field distribution of the resonant mode:

$\frac{\Delta f}{f_{0}}=\left(\varepsilon_{r}^{\prime}-1\right) \frac{V_{S}}{2 V_{\text {eff }}}$ 
Moreover, the dielectric losses can be calculated from the change in the inverse resonant quality:

$\Delta\left(\frac{1}{\mathrm{Q}}\right)=\varepsilon_{\mathrm{r}}^{\prime \prime} \frac{V_{\mathrm{S}}}{V_{\text {eff }}}$

These equations can only be applied to a, corresponding to the MCP theory, ideal system. If these preconditions are not fulfilled, various correction terms have to be applied for a correct determination of the dielectric properties. For the setup described in this work, especially the following three points should be considered [4]:

- Deviations of the field distribution of the real setup compared to a perfectly cylindrical resonator lead to a wrong $V_{\text {eff. }}$. These are caused, e.g., by the openings required for heating or by the quartz glass tubes used to place the material sample. However, the correct determination of $V_{\text {eff }}$ can be obtained by a simulation of the field distribution.

- Depolarization effects can lead to a field weakening inside the sample. In consequence, the resonant parameters are influenced to a smaller extent by the sample than assumed in the simplified theory. This effect can be corrected if the sample geometry is known.

- To determine the effective dielectric properties of porous samples, such as powders, the portion of the different components has to be considered by a mixing model. In the literature a large number of various models is described (e.g. Wiener, Looyenga, ...). Which of these can be applied to the measured material can be analyzed almost exclusively by further measurements.

\section{Results}

In addition to solid oxide fuels cells, ceria is frequently used for three-way catalytic converters in automobiles. In this application, ceria serves as an oxygen storage material. Since its dielectric properties depend on its oxygen stoichiometry, the investigation of ceria using MCP is particularly important for RF-based state diagnosis. Tab. 1 shows the results for a ceria powder sample in the resonator at room temperature. The calculation of $\varepsilon_{\mathrm{r}}{ }^{\prime}$ with both approaches clearly shows that the extended approach is necessary to calculate the known properties of ceria. The extended method uses a simulation-determined $V_{\text {eff }}$, the mixture model according to Looyenga and considers the depolarization effect.

Tab. 1: Permittivity $\varepsilon_{r}{ }^{\prime}$ of ceria at $25{ }^{\circ} \mathrm{C}$ and $20 \% \mathrm{O}_{2}$

\begin{tabular}{|c|c|c|c|}
\hline mode & $\begin{array}{c}\text { simplified } \\
\text { theory }\end{array}$ & $\begin{array}{c}\text { extended } \\
\text { theory }\end{array}$ & literature ${ }^{[5]}$ \\
\hline TM$_{010}$ & 3.95 & 22.6 & \multirow{2}{*}{23} \\
\hline TM $_{020}$ & 9.92 & 23.6 & \\
\hline
\end{tabular}

The determination of an applicable mixture model for an investigated material represents a great effort. It depends not only on the material itself, but also on other parameters such as its particle size distribution.

The mixture model for a soot loaded particulate filter, for example, can be determined by measuring different mixtures of synthetic soot (PrintexU) and cordierite in the resonator setup.

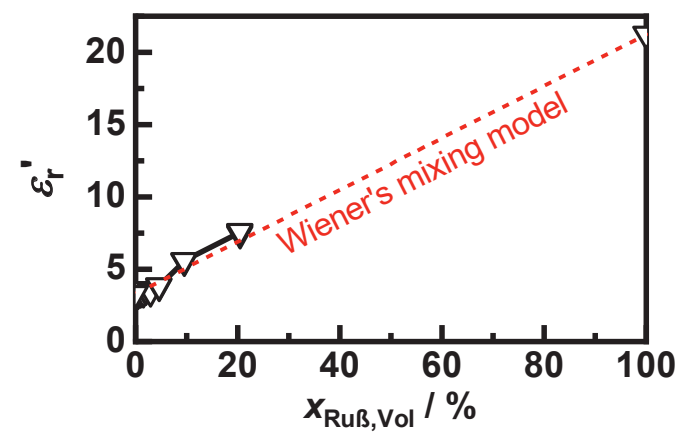

Fig. 2. Permittivity of different PrintexU - cordierite mixtures at room temperature.

The measured permittivity in Fig. 2 show a behavior equivalent to Wiener's mixing model. Thus, this model can be used in further measurements to determine directly the permittivity of pure soot.

\section{References}

[1] R. Moos, Microwave-Based Catalyst State Diagnosis - State of the Art and Future Perspectives, SAE Int. J. Engines 8, 1240-1245 (2015); doi: 10.4271/2015-01-1042.

[2] R. Moos, et al., Automotive Catalyst State Diagnosis Using Microwaves, Oil Gas Sci. Technol. 70, 55-65 (2015); doi: 10.2516/ogst/2013203.

[3] M. Dietrich, et al., A Laboratory Test Setup for in Situ Measurements of the Dielectric Properties of Catalyst Powder Samples under Reaction Conditions by Microwave Cavity Perturbation: Set up and Initial Tests, Sensors 14, 16856-16868 (2014); doi: 10.3390/s140916856.

[4] C. Steiner, et al., Determination of the Dielectric Properties of Storage Materials for Exhaust Gas Aftertreatment Using the Microwave Cavity Perturbation Method, Sensors 20, 6024 (2020); doi: $10.3390 / \mathrm{s} 20216024$.

[5] N. I. Santha, et al., Effect of Doping on the Dielectric Properties of Cerium Oxide in the Microwave and Far-Infrared Frequency Range, J. Am. Ceram. Soc. 87, 1233-1237 (2004); doi: 10.1111/j.11512916.2004.tb07717_33.x.

\section{Funding}

This research was funded by the Deutsche Forschungsgemeinschaft DFG, (MO 1060/29-1 and FR 1301/23-1) and the Bayerische Forschungsstiftung BFS (AZ-1288-17). 